\title{
Topological arguments for an elliptic equation involving the fractional Laplacian
}

\author{
Mohammed Ali Al-Ghamdi ${ }^{*}$, Wael Abdelhedi ${ }^{2}$ and Hichem Chtioui ${ }^{1}$
}

${ }^{\text {*Correspondence: }}$

MGHAMDI3@kau.edu.sa

'Department of Mathematics, King

Abdulaziz University, Jeddah, Saudi

Arabia

Full list of author information is

available at the end of the article

\begin{abstract}
In this paper, we consider a fractional Nirenberg-type problem involving $\sigma$-exponent of the Laplacian on the standard $n$-dimensional spheres $\mathbf{S}^{n}$. Using an algebraic topological method and the theory of the critical points at infinity, we provide a variety of classes of functions that can be realized as the $\sigma$-curvature on n-dimensional sphere.
\end{abstract}

MSC: 35M30; 35Q74; 74A15; 74A60; 74M25

Keywords: fractional Laplacian; critical exponent; critical points at infinity; Nirenberg problem

\section{Introduction and main results}

Let $\left(\mathbf{S}^{n}, g\right)$ be the standard sphere of dimension $n, n \geq 2$ and $K$ be a positive function on $\mathbf{S}^{n}$. The classical Nirenberg problem is the following: can one change the original metric $g$ conformally into a new metric $\tilde{g}$ with prescribed scalar curvature equal to $K$ for $n \geq$ 3 (prescribed Gaussian curvature for $n=2$ ). This problem is equivalent to solving the following nonlinear elliptic equations:

$$
\begin{aligned}
& -\Delta_{g}(u)+1=K e^{2 u} \quad \text { on } \mathbf{S}^{2} \\
& L_{g}(u)=K u^{\frac{n+2}{n-2}} \quad \text { on } \mathbf{S}^{n}, n \geq 3
\end{aligned}
$$

where $L_{g}:=-c_{n} \Delta_{g}+R_{g}$ be the conformal Laplacian, $\Delta_{g}$ is the Laplace-Beltrami operator, $c_{n}=4 \frac{n-1}{n-2}$ and $R_{g}$ is the scalar curvature associated to the metric $g$.

Recently, several studies have been performed for classical elliptic equations similar to (2) and (1) but with the fractional conformal Laplacians instead of the Laplacian. This operator is introduced first in [1] where Graham, Jenne, Mason, and Sparling constructed a sequence of conformally covariant elliptic operators $P_{k}^{g}$, on Riemannian manifolds $(M, g)$ for all positive integers $k$ if $n$ is odd, and for $k \in\{1, \ldots, n / 2\}$ if $n$ is even. Moreover, $P_{1}^{g}$ is the well-known conformal Laplacian $L_{g}$ used in (2), and $P_{2}^{g}$ is the well-known Paneitz operator. Up to positive constants $P_{1}^{g}(1)$ is the scalar curvature $R_{g}$ associated to $g$ and $P_{2}^{g}(1)$ is the $Q$-curvature. Prescribing scalar curvature and Q-curvature problems on $\mathbf{S}^{n}$ was studied extensively in last years, see for example [2-10]; see also [11-19], and the references therein.

In [20], Chang and Gonzalez generalize the above construction, using the works of Graham-Zworski [21] and Caffarelli-Silvestre [22] for factional Laplacian on the Euclidean

\section{Springer}

(c) 2014 Al-Ghamdi et al.; licensee Springer. This is an Open Access article distributed under the terms of the Creative Commons Attribution License (http://creativecommons.org/licenses/by/2.0), which permits unrestricted use, distribution, and reproduction in any medium, provided the original work is properly credited. 
space $\mathbf{R}^{n}$, to define conformally invariant operators $P_{\sigma}^{g}$ of non-integer order $\sigma$. These lead naturally to a fractional-order curvature $R_{g}:=P_{\sigma}^{g}(1)$, which will be called here $\sigma$-curvature. For the $\sigma$-curvatures on general manifolds we refer to [20,21,23], and references therein. Corresponding to the Yamabe problem, fractional Yamabe problems for $\sigma$-curvatures are studied in [23, 24], and [25] and fractional Yamabe flows on $\mathbf{S}^{n}$ are studied in [26].

In this paper, we restrict our attention to the case $\sigma=\frac{1}{2}$. As in the Nirenberg problem associated to $P_{1}^{g}$ and $P_{2}^{g}$, the question of prescribing $\frac{1}{2}$-curvature can be formulated as a Nirenberg-type problem involving the square root of the Laplacian as follows: given a positive function $K$ defined on $\left(\mathbf{S}^{n}, g\right)$, we ask whether there exists a metric $\tilde{g}$ conformally equivalent to $g$ such that the $\frac{1}{2}$-curvature is equal to $K$. This problem can be expressed as finding the solution of the following nonlinear equation with critical exponent,

$$
P_{\frac{1}{2}} u=c_{n} K u^{\frac{n+1}{n-1}}, \quad u>0, \text { on } S^{n}
$$

where

$$
P_{\frac{1}{2}}=\frac{\Gamma(B+1)}{\Gamma(B)}, \quad B=\sqrt{-\Delta_{g}+\left(\frac{n-1}{2}\right)^{2}},
$$

$c_{n}=P_{\frac{1}{2}}(1), \Gamma$ is the gamma function and $\Delta_{g}$ is the Laplace-Beltrami operator on $\left(\mathbf{S}^{n}, g\right)$. The operator $P_{\frac{1}{2}}$ can be seen more concretely on $\mathbf{R}^{n}$ using stereographic projection. The stereographic projection from $\mathbf{S}^{n} \backslash N$ to $\mathbf{R}^{n}$ is the inverse of $F: \mathbf{R}^{n} \rightarrow \mathbf{S}^{n} \backslash N$ defined by

$$
F(x)=\left(\frac{2 x}{1+|x|^{2}}, \frac{|x|^{2}-1}{|x|^{2}+1}\right)
$$

where $N$ is the north pole of $\mathbf{S}^{n}$. Then, for all $f \in C^{\infty}\left(\mathbf{S}^{n}\right)$, we have

$$
\left(P_{\frac{1}{2}}(f)\right) \circ F=\left(\frac{2}{1+|x|^{2}}\right)^{\frac{-(n+1)}{2}}(-\Delta)^{1 / 2}\left(\left(\frac{2}{1+|x|^{2}}\right)^{\frac{n+1}{2}}(f \circ F)\right),
$$

where $(-\Delta)^{1 / 2}$ is the fractional Laplacian operator (see, e.g., p.117 of [27]).

A sufficient condition was found recently in [28] for the two dimensional sphere, it is an Euler-Hopf-type criterium, namely the authors proved the following existence result.

Theorem 1.1 [28] Let $K$ be a positive function satisfying the following (nd) condition:

(nd): Assume that $K$ is a smooth function on $S^{2}$ having only non-degenerate critical points with

$$
\Delta K(y) \neq 0 \quad \text { whenever } \nabla K(y)=0 .
$$

Let

$$
\mathcal{K}=\left\{y \in S^{2}, \nabla K(y)=0\right\} \text { and } \mathcal{K}^{+}=\{y \in \mathcal{K},-\Delta K(y)>0\} .
$$

If

(R) $\sum_{y \in \mathcal{K}^{+}}(-1)^{2-\operatorname{ind}(K, y)} \neq 1$,

then (3) has at least one solution. Here, $\operatorname{ind}(K, y)$ is the Morse index of $K$ at $y$. 
A natural question which arises when looking to the above result is what happens if the total sum in $\left(R_{1}\right)$ is equal to 1 , but a partial one is not. Under which condition can one use this partial sum to derive an existence result? Our aim in the first part of this paper is to give a partial answer to this question and to prove new existence criterium which generalizes the index-count formula in $\left(\mathrm{R}_{1}\right)$ and recover the previous existence result obtained in [28]. To state our result, we set the following notations. We order the $K(y), y \in \mathcal{K}$. Without loss of generality, we assume that the elements of $\mathcal{K}$ are $y_{0}, y_{1}, \ldots, y_{l}$ with

$$
K\left(y_{0}\right) \geq K\left(y_{1}\right) \geq \cdots \geq K\left(y_{l}\right) .
$$

Let $k$ be an index, $0 \leq k \leq l$. We say that $k \in\left(\mathbf{A}_{1}\right)$ if it satisfies the following:

$\left(\mathbf{A}_{1}\right)$ If there exists an index $j>k$ such that $y_{j} \in \mathcal{K}^{+}$, we have

$$
\frac{1}{K\left(y_{j}\right)}>\frac{1}{K\left(y_{k}\right)}+\frac{1}{K\left(y_{0}\right)} \text {. }
$$

Now, we are ready to state our first main result.

Theorem 1.2 Let $K$ be a positive function on $\mathbf{S}^{2}$ and satisfying (nd) condition. If

$$
\underset{k \in\left(\mathbf{A}_{1}\right)}{\operatorname{Max}}\left|1-\sum_{\substack{y_{j} \in \mathcal{K}^{+} \\ 0 \leq j \leq k}}(-1)^{\operatorname{ind}\left(K, y_{j}\right)}\right| \neq 0,
$$

then (3) has at least one solution. Moreover, for generic $K$ we have

$$
\sharp S \geq \operatorname{Max}_{k \in\left(\mathbf{A}_{1}\right)}\left|1-\sum_{\substack{y_{j} \in \mathcal{K}^{+} \\ 0 \leq j \leq k}}(-1)^{\operatorname{ind}\left(K, y_{j}\right)}\right|,
$$

where $S$ denotes the set of solutions of (3).

Observe that the index $l \in\left(\mathbf{A}_{1}\right)$. It follows that the above mentioned result (Theorem 1.1) is a corollary of our theorem. Actually, we will give in Remark 3.1 a situation where Theorem 1.1 does not give a result, while by Theorem 1.2, we derive the existence of many solutions.

Second, we consider the case $n \geq 3$ and $K$ close to a constant, i.e. $K$ of the from $K=$ $K_{\varepsilon}=1+\varepsilon K_{0}$, where $K_{0} \in C^{2}\left(\mathbf{S}^{n}\right)$ and $|\varepsilon|$ small. In order to state our result, we need the following assumption. We say that an integer $k \in\left(\mathbf{A}_{2}\right)$ if it satisfies the following:

$\left(\mathbf{A}_{2}\right)$ For any $y \in \mathcal{K}_{\varepsilon}{ }^{+}$, we have $n-\operatorname{ind}\left(K_{\varepsilon}, y\right) \neq k+1$.

We shall prove the following result.

Theorem 1.3 Assume that $K_{\varepsilon}$ is a Morse function on $\mathbf{S}^{n}, n \geq 3$ and satisfying (nd) condition. If,

$$
\operatorname{Max}_{k \in\left(\mathbf{A}_{2}\right)}\left|1-\sum_{\substack{y \in \mathcal{K}_{\varepsilon}{ }^{+} \\ n-\operatorname{ind}\left(K_{\varepsilon}, y\right) \leq k}}(-1)^{n-\operatorname{ind}\left(K_{\varepsilon}, y\right)}\right| \neq 0,
$$

then, for $|\varepsilon|$ sufficiently small, there exists a solution to the problem (3). 
Observe that $n \in\left(\mathbf{A}_{2}\right)$. We therefore have the following corollary.

Corollary 1.4 Let $K_{\varepsilon}$ be a Morse function with (nd) condition. If

$$
\sum_{y \in \mathcal{K}_{\varepsilon}{ }^{+}}(-1)^{n-\operatorname{ind}\left(K_{\varepsilon}, y\right)} \neq 1
$$

then for $|\varepsilon|$ sufficiently small, (3) has at least one solution.

The rest of this paper is organized as follows: we first recall some known facts about the variational structure of problem (3) and the associated critical points at infinity. In Section 3, we give the proofs of our main results and in Section 4, we give a more general result for $\sigma \in(0,1)$ and $2 \leq n<2+2 \sigma$.

\section{Preliminary results}

\subsection{The variational structure}

First, we recall that $u \in H^{\frac{1}{2}}\left(\mathbf{S}^{n}\right)$ is a solution of (3) if the identity

$$
\int_{\mathbf{S}^{n}} P_{\frac{1}{2}} u \varphi d x=c_{n} \int_{\mathbf{S}^{n}} K u^{\frac{n+1}{n-1}} \varphi d x
$$

holds for all $\varphi \in H^{\frac{1}{2}}\left(\mathbf{S}^{n}\right)$, where $H^{\frac{1}{2}}\left(\mathbf{S}^{n}\right)$ denotes the closure of $C^{\infty}\left(\mathbf{S}^{n}\right)$ under the norm

$$
\|u\|_{H^{\frac{1}{2}\left(\mathbf{S}^{n}\right)}}=\left(\int_{\mathbf{S}^{n}} P_{\frac{1}{2}} u u\right)^{1 / 2} .
$$

Observe also that for $u \in H^{\frac{1}{2}}\left(\mathbf{S}^{n}\right)$, we have $u^{\frac{n+1}{n-1}} \in L^{\frac{2 n}{n+1}}\left(\mathbf{S}^{n}\right) \hookrightarrow H^{-\frac{1}{2}}\left(\mathbf{S}^{n}\right)$. We associate to problem (3), the functional

$$
I(u)=\frac{1}{2} \int_{\mathbf{S}^{n}} u P_{\frac{1}{2}} u-\frac{n-1}{2 n} \int_{\mathbf{S}^{n}} K u^{\frac{2 n}{n-1}}
$$

defined in $H^{\frac{1}{2}}\left(\mathbf{S}^{n}\right)$.

Motivated by the work of Caffarelli and Silvestre [22], several authors have considered an equivalent definition of the operator $P_{\frac{1}{2}}$ by means of an auxiliary variable; see [22] (see also [29-32], and [33]). In fact, we realize problem (3), through a localization method introduced by Caffarelli and Silvestre on the Euclidean space $\mathbf{R}^{n}$, through which (3) is connected to a degenerate elliptic differential equation in one dimension higher by a Dirichlet to Neumann map. This provides a good variational structure to the problem. By studying this problem with classical local techniques, we establish existence of positive solutions. Here the Sobolev trace embedding comes into play, and its critical exponent $2^{*}=\frac{2 n}{n-1}$.

Namely, let $D_{n}=\mathbf{S}^{n} \times[0, \infty)$. Given $u \in H^{\frac{1}{2}}\left(\mathbf{S}^{n}\right)$, we define its harmonic extension $U=$ $E_{\sigma}(u)$ to $D_{n}$ as the solution to the problem

$$
\left\{\begin{array}{l}
-\Delta U=0 \quad \text { in } D_{n} \\
U=u \text { on } \mathbf{S}^{n} \times\{t=0\} .
\end{array}\right.
$$


The extension belongs to the space $H^{1}\left(D_{n}\right)$ defined as the completion of $C^{\infty}\left(D_{n}\right)$ with the norm

$$
\|U\|_{H^{1}\left(D_{n}\right)}=\left(\int_{D_{n}}|\nabla U|^{2} d x d t\right)^{1 / 2} .
$$

Observe that this extension is an isometry in the sense that

$$
\|U\|_{H^{1}\left(D_{n}\right)}=\|u\|_{H^{\frac{1}{2}\left(\mathbf{S}^{n}\right)}}, \quad \forall u \in H^{\frac{1}{2}}\left(\mathbf{S}^{n}\right) .
$$

Moreover, for any $\varphi \in H^{1}\left(D_{n}\right)$, we have the following trace inequality:

$$
\|\varphi\|_{H^{1}\left(D_{n}\right)} \geq\|\varphi(\cdot, 0)\|_{H^{\frac{1}{2}\left(\mathbf{S}^{n}\right)}} \cdot
$$

The relevance of the extension function $U=E_{\sigma}(u)$ is that it is related to the fractional Laplacian of the original function $u$ through the formula

$$
-\lim _{t \rightarrow 0^{+}} \frac{\partial U}{\partial t}(x, t)=P_{\frac{1}{2}} u(x)
$$

Thus, we can reformulate (3) as follows:

$$
\left\{\begin{array}{l}
-\Delta U(x, t)=0 \text { and } \quad U>0 \text { in } D_{n} \\
-\lim _{t \rightarrow 0^{+}} \frac{\partial U}{\partial t}(x, t)=K U^{\frac{n+1}{n-1}}(x, 0) \text { on } \mathbf{S}^{n} \times\{0\}
\end{array}\right.
$$

The functional associated to (13) is given by

$$
I_{1}(U)=\frac{1}{2} \int_{D_{n}}|\nabla U|^{2} d x d t-\frac{n-1}{2 n} \int_{S^{n}} K U^{\frac{2 n}{n-1}} d x,
$$

defined in $H^{1}\left(D_{n}\right)$.

Notice that critical points of $I_{1}$ in $H^{1}\left(D_{n}\right)$ correspond to critical points of $I$ in $H^{\frac{1}{2}}\left(\mathbf{S}^{n}\right)$. That is, if $U=E_{\sigma}(u)$ satisfies (13), then $u$ will be a solution of problem (3).

Let also define the functional

$$
J(U)=\frac{\|U\|_{H^{1}\left(D_{n}\right)}^{2}}{\left(\int_{\mathbf{S}^{n}} K U^{\frac{2 n}{n-1}} d x\right)^{\frac{n-1}{n}}},
$$

defined on $\Sigma$ the unit sphere of $H^{1}\left(D_{n}\right)$. We set, $\Sigma^{+}=\{U \in \Sigma / U \geq 0\}$. Problem (3) will be reduced to finding the critical points of $J$ subjected to the constraint $U \in \Sigma^{+}$. The exponent $\frac{2 n}{n-1}$ is critical for the Sobolev trace embedding $H^{1}\left(D_{n}\right) \rightarrow L^{q}\left(\mathbf{S}^{n}\right)$. This embedding being continuous and not compact, the functional $J$ does not satisfy the Palais-Smale condition, which leads to the failure of the standard critical point theory. This means that there exist sequences along which $J$ is bounded, its gradient goes to zero and which do not converge. The analysis of sequences failing PS condition can be analyzed along the ideas introduced in [2] and [34]. In order to describe such a characterization in our case, we need to introduce some notations. 
For $a \in \partial \mathbf{R}_{+}^{n+1}$ and $\lambda>0$, define the function:

$$
\tilde{\delta}_{a, \lambda}(x)=\bar{c} \frac{\lambda^{\frac{n-1}{2}}}{\left(\left(1+\lambda x_{n+1}\right)^{2}+\lambda^{2}\left|x^{\prime}-a^{\prime}\right|^{2}\right)^{\frac{n-1}{2}}},
$$

where $x \in \mathbf{R}_{+}^{n+1}$, and $\bar{c}$ is chosen such that $\tilde{\delta}_{a, \lambda}$ satisfies the following equation:

$$
\begin{cases}\Delta U=0 \quad \text { and } & U>0 \quad \text { in } \mathbf{R}_{+}^{n+1} \\ -\frac{\partial U}{\partial x_{n+1}}=U^{\frac{n+1}{n-1}} & \text { on } \partial \mathbf{R}_{+}^{n+1} .\end{cases}
$$

Set

$$
\delta_{a, \lambda}=i^{-1}\left(\tilde{\delta}_{a, \lambda}\right)
$$

where $i$ is an isometry from $H^{1}\left(D_{n}\right)$ to $D^{1,2}\left(\mathbf{R}_{+}^{n+1}\right)$; the completion of $C_{c}^{\infty}\left(\overline{\mathbf{R}_{+}^{n+1}}\right)$, with respect to the Dirichlet norm.

For $\varepsilon>0$ and $p \in \mathbf{N}^{*}$, we define

$$
V(p, \varepsilon)=\left\{\begin{array}{l}
U \in \Sigma \text { s.t. } \exists a_{1}, \ldots, a_{p} \in S^{n}, \exists \alpha_{1}, \ldots, \alpha_{p}>0 \text { and } \\
\exists \lambda_{1}, \ldots, \lambda_{p}>\varepsilon^{-1} \text { with }\left\|U-\sum_{i=1}^{p} \alpha_{i} \delta_{a_{i}, \lambda_{i}}\right\|<\varepsilon, \varepsilon_{i j}<\varepsilon, \forall i \neq j, \text { and } \\
\left|J(U)^{\frac{n}{n-1}} \alpha_{i}^{\frac{2}{n-1}} K\left(a_{i}\right)-1\right|<\varepsilon, \forall i, j=1, \ldots, p,
\end{array}\right.
$$

where

$$
\varepsilon_{i j}=\left(\frac{\lambda_{i}}{\lambda_{j}}+\frac{\lambda_{j}}{\lambda_{i}}+\lambda_{i} \lambda_{j}\left|a_{i}-a_{j}\right|^{2}\right)^{\frac{1-n}{2}} .
$$

If $U$ is a function in $V(p, \varepsilon)$, one can find an optimal representation, following the ideas introduced in [35], namely we have:

Lemma 2.1 For any $p \in \mathbf{N}^{*}$, there is $\varepsilon_{p}>0$ such that if $\varepsilon \leq \varepsilon_{p}$ and $U \in V(p, \varepsilon)$, then the minimization problem

$$
\min \left\{\left\|U-\sum_{i=1}^{p} \alpha_{i} \delta_{\left(a_{i}, \lambda_{i}\right)}\right\|, \alpha_{i}>0, \lambda_{i}>0, a_{i} \in \mathbf{S}^{n}\right\}
$$

has a unique solution $(\bar{\alpha}, \bar{\lambda}, \bar{a})$. Thus, we can write $U$ as follows:

$$
U=\sum_{i=1}^{p} \alpha_{i} \delta_{\left(a_{i}, \lambda_{i}\right)}+v
$$

where $v$ belongs to $H^{1}\left(D_{n}\right)$ and satisfies the following condition:

$\left(\mathrm{V}_{0}\right):\left\langle v, \varphi_{i}\right\rangle=0$ for $i=1, \ldots, p$, and $\varphi_{i}=\delta_{i}, \partial \delta_{i} / \partial \lambda_{i}, \partial \delta_{i} / \partial a_{i}$, where $\delta_{i}=\delta_{a_{i}, \lambda_{i}}$ and $\langle\cdot, \cdot\rangle$ denote the scalar product defined on $H^{1}\left(D_{n}\right)$ by

$$
\langle U, V\rangle=\int_{D_{n}} \nabla U \nabla V d x d t
$$


The failure of the Palais-Smale condition can be characterized taking into account the uniqueness result of the corresponding problem at infinity; see e.g. Li-Zhu [36] following the ideas introduced in $[34,37]$ as follows.

Proposition 2.2 Assume that (3) has no solution and let $\left(U_{k}\right) \subset \Sigma^{+}$be a sequence satisfying $J\left(U_{k}\right) \rightarrow c$, a positive number and $\partial J\left(U_{k}\right) \rightarrow 0$. Then there exist an integer $p \geq 1$, a positive sequence $\left(\varepsilon_{k}\right)_{k}\left(\varepsilon_{k} \rightarrow 0\right)$ and an extracted subsequence of $\left(U_{k}\right)$, again denoted $U_{k}$ such that $U_{k} \in V\left(p, \varepsilon_{k}\right)$.

Following Bahri [35, 38], we set the following definition and notation.

Definition 2.3 A critical point at infinity of $J$ on $\Sigma^{+}$is a limit of a flow line $U(s)$ of the equation:

$$
\left\{\begin{array}{l}
\frac{\partial U}{\partial s}=-J(U), \\
U(0)=U_{0},
\end{array}\right.
$$

such that $U(s)$ remains in $V(p, \varepsilon(s))$ for $s \geq s_{0}$.

Using Lemma 2.1, $U(s)$ can be written as

$$
U(s)=\sum_{i=1}^{p} \alpha_{i(s)} \delta_{\left(a_{i}(s), \lambda_{i}(s)\right)}+v(s) .
$$

Denoting $a_{i}:=\lim _{s \rightarrow \infty} a_{i}(s)$ and $\alpha_{i}=\lim _{s \rightarrow \infty} \alpha_{i}(s)$, we denote by

$$
\left(a_{1}, \ldots, a_{p}\right)_{\infty} \quad \text { or } \quad \sum_{i=1}^{p} \alpha_{i} \delta_{\left(a_{i}, \infty\right)}
$$

such a critical point at infinity.

The following propositions characterize the critical points at infinity of the associated variational problem.

Proposition 2.4 Assume that $n=2$. Under the (nd) condition, the only critical points at infinity of the functional $J$ are:

$$
(y)_{\infty}:=\frac{1}{K(y)^{\frac{1}{2}}} \tilde{\delta}_{(y, \infty)}, \quad y \in \mathcal{K}^{+} .
$$

Such a critical point at infinity has a Morse index equal to $i(y)_{\infty}:=2-\operatorname{ind}(K, y)$. Its level is $c_{\infty}(y):=\widetilde{S}_{2} \frac{1}{K(y)^{\frac{1}{2}}}$, where $\widetilde{S}_{2}$ is the best constant of Sobolev.

Proof See Corollary 4.4 of [28].

Arguing as in [28], we have the following.

Proposition 2.5 Assume that $n \geq 3$ and assume that $J$ has no critical point in $\Sigma^{+}$. Then the only critical points at infinity of $J$ in $V(1, \varepsilon)$ are

$$
(y)_{\infty}:=\frac{1}{K(y)^{\frac{n-1}{n}}} \tilde{\delta}_{(y, \infty)}, \quad y \in \mathcal{K}^{+} .
$$


The Morse index of $(y)_{\infty}$ is equal to $i(y)_{\infty}:=n-\operatorname{ind}(K, y)$. Its level is $c_{\infty}(y):=\widetilde{S}_{n} \frac{1}{K(y)^{\frac{n-1}{n}}}$. Here $\widetilde{S}_{n}$ is the best constant of Sobolev.

\subsection{The unstable manifolds of critical points at infinity}

At the beginning of this subsection, we give some basic definitions with will allow us to describe the unstable manifolds of the critical points at infinity in $V(1, \varepsilon)$.

Definition 2.6 Let $K: S^{n} \rightarrow \mathbf{R}$ be a $C^{2}$ Morse function and let $\mathcal{K}$ the set of critical points of $K$. If $y \in \mathcal{K}$, let $W_{s}(y)$ designate its stable manifold and $W_{u}(y)$ designate its unstable manifold. We have

$$
\operatorname{dim} W_{u}(y)=\operatorname{ind}(K, y) \quad \text { and } \quad \operatorname{dim} W_{s}(y)=n-\operatorname{ind}(K, y) .
$$

It is convenient to specify that the notations of stable or unstable manifolds, of flow lines, all are relative to the $C^{1}$ vector field $(-\partial K)$, with respect to the standard Riemannian structure on $S^{n}$. Recall the following generic hypothesis:

All stable and unstable manifolds intersect transversely and all such intersections are smooth regularly embedded submanifolds of $S^{n}$.

Definition 2.7 Let $y, z \in \mathcal{K} . z$ is said to be dominated by $y$, if

$$
W_{u}(y) \cap W_{s}(z) \neq \emptyset,
$$

then there exists (at least) a flow line of ( $-\partial K)$ descending from y to $z$. Using the dimension argument and the fact that both of $W_{u}(y)$ and $W_{s}(z)$ are invariant under the action of the flow generated by $(-\partial K)$, it is easy to see that

$$
\text { if } W_{u}(y) \cap W_{s}(z) \neq \emptyset \text {, then } \operatorname{ind}(K, y) \geq \operatorname{ind}(K, z)+1 \text {. }
$$

Definition 2.8 Let $y \in \mathcal{K}^{+}=\{y \in \mathcal{K},-\Delta K(y)>0\}$. In $V(1, \varepsilon)$, the unstable manifold at infinity $W_{u}^{\infty}(y)_{\infty}$ for the critical point at infinity $(y)_{\infty}$, along the flow lines of $-\partial J$ is defined and identified by the unstable manifold $\widetilde{W}_{u}(y)$ of the critical point $y$ of the function $\frac{1}{K}$, along the flow lines of $-\partial\left(\frac{1}{K}\right)$ multiplied by a factor corresponding to the concentration $\lambda$. Precisely in $V(1, \varepsilon)$, the $W_{u}^{\infty}(y)_{\infty}$ have the following description:

$$
W_{u}^{\infty}(y)_{\infty} \cap V(1, \varepsilon)=\left\{\frac{1}{\widetilde{S}_{n}} \tilde{\delta}_{(a, \lambda)}, a \in \widetilde{W}_{u}(y)\right\}
$$

where $\lambda$ is a fixed constant large enough.

Remark 2.9 Observe that $\widetilde{W}_{u}(y)$ correspond to $W_{s}(y)$, the stable manifold of the critical point $y$ along the flow lines of $(-\partial K)$. Therefore it is easy to see that if $W_{u}^{\infty}(y)_{\infty} \subset V(1, \varepsilon)$, then $W_{u}^{\infty}(y)_{\infty}$ behaves as $W_{s}(y)$.

The following lemma gives a sufficient condition to ensure that $W_{u}^{\infty}(y)_{\infty}$ is included in $V(1, \varepsilon)$. 
Lemma 2.10 Let $y \in \mathcal{K}^{+}$.If

$$
W_{s}(y) \cap W_{u}(z)=\emptyset, \forall z \in \mathcal{K} \backslash \mathcal{K}^{+} \text {, then } W_{u}^{\infty}(y)_{\infty} \text { diffeomorphic to } W_{s}(y) \text {. }
$$

Proof It follows from [28]. The idea is that a flow line in $W_{u}^{\infty}(y)_{\infty}$ cannot go out from $V(1, \varepsilon)$ unless the concentration point $a(s)$ of the flow line nearby a critical point $z$ of $K$ with $-\Delta K(z)<0$ (see proof of Proposition 4.3 of [28]), therefore it is the case when the critical point $y$ is dominated by $z \in \mathcal{K} \backslash \mathcal{K}^{+}$. Hence under the condition of the lemma such a situation cannot occur, it follows that every flow line in $W_{u}^{\infty}(y)_{\infty}$ is indeed in $V(1, \varepsilon)$ and we then conclude to the result of the lemma using Remark 2.9.

\section{Proof of results}

This section is devoted to the proofs of the main results of this paper. Our proofs uses algebraic topological arguments and the tools of the theory of critical points at infinity; see [38]. In our case, the space of variation is contractible and has no topology. However, due to the non-compactness of the problem, there are critical points at infinity whose topological contribution can be computed; see [28]. The main idea is to use the difference of topology of the critical points at infinity between the level sets of the associated EulerLagrange functional $J$, and the main issue is under our conditions on $K$, there remains some difference of topology which is not due to the critical points at infinity but due to the existence of solution of (3).

\subsection{Proof of Theorem 1.2}

We denote by $y_{0}, y_{1}, \ldots, y_{l}$ the elements of $\mathcal{K}$. We order the $K\left(y_{i}\right)^{\prime}$ 's, $y_{i} \in \mathcal{K}$. Without loss of generality, we assume that

$$
K\left(y_{0}\right) \geq K\left(y_{1}\right) \geq \cdots \geq K\left(y_{l}\right) .
$$

Recall that, see Proposition 2.4, the critical points at infinity are in one to one correspondence with the elements $y_{i}$ of the sets $\mathcal{K}^{+}$. Like a usual critical point, it is associated to a critical point at infinity $\left(y_{i}\right)_{\infty}$, stable and unstable manifolds, $W_{s}^{\infty}\left(y_{i}\right)_{\infty}$ and $W_{u}^{\infty}\left(y_{i}\right)_{\infty}$. Let $0 \leq k_{0} \leq l$ be an index such that

$$
\operatorname{Max}_{k \in\left(\mathbf{A}_{1}\right)}\left|1-\sum_{\substack{y_{j} \in \mathcal{K}^{+} \\ 0 \leq j \leq k}}(-1)^{\operatorname{ind}\left(K, y_{j}\right)}\right|
$$

is achieved. Let

$$
c_{1}=\frac{1}{K\left(y_{0}\right)}+\frac{1}{K\left(y_{k_{0}}\right)} \text {. }
$$

Since $k_{0}$ satisfies $\left(\mathbf{A}_{1}\right)$, we can find $\varepsilon>0$ satisfying the following:

For any $j>k_{0}$ such that $y_{j} \in \mathcal{K}^{+}$, we have

$$
c_{\infty}\left(y_{j}\right)=\frac{1}{K\left(y_{j}\right)}>c_{1}+\varepsilon .
$$


Therefore the only critical points at infinity under the level $c_{1}+\varepsilon$ are:

$$
\left(y_{j}\right)_{\infty}, \quad y_{j} \in \mathcal{K}^{+} \text {such that } 0 \leq j \leq k_{0}
$$

Let

$$
X_{k_{0}}^{\infty}:=\bigcup_{y_{j} \in \mathcal{K}^{+}, 0 \leq j \leq k_{0}} W_{u}^{\infty}\left(y_{j}\right)_{\infty} .
$$

Given $c \in \mathbf{R}$, we set

$$
J^{c}:=\left\{u \in \Sigma^{+}, J(u) \leq c\right\} .
$$

We claim that

$$
X_{k_{0}}^{\infty} \text { is contractible in } J^{c_{1}+\varepsilon} \text {. }
$$

Indeed, let

$$
X_{k_{0}}:=\bigcup_{y_{j} \in \mathcal{K}^{+}, 0 \leq j \leq k_{0}} W_{s}\left(y_{j}\right),
$$

where $W_{s}\left(y_{j}\right)$ is the stable manifold of $y_{j}$ along the flow lines of $(-\partial K)$. Observe that for any $y \in \mathcal{K}^{+}$, we have ind $(K, y)=1$ or 2 , thus from (16), $y$ cannot be dominated through the flow lines of $(-\partial K)$ only by critical points $y^{\prime}$ of $K$ such that $\operatorname{ind}\left(K, y^{\prime}\right)=2$, thus it satisfies $-\Delta K\left(y^{\prime}\right)>0$. Therefore we obtain

$$
W_{s}(y) \cap W_{u}(z)=\emptyset, \quad \forall z \in \mathcal{K} \backslash \mathcal{K}^{+}
$$

Using now Lemma 2.10, we derive that

$$
X_{k_{0}}^{\infty} \text { is diffeomorphic to } X_{k_{0}} \text {. }
$$

More precisely,

$$
X_{k_{0}}^{\infty}=\left\{\frac{1}{\widetilde{S}_{2}} \tilde{\delta}_{(x, \lambda)}, x \in X_{k_{0}}\right\},
$$

where $\lambda$ is a fixed constant large enough and $\widetilde{S}_{2}$ is the best constant of Sobolev.

Now, let

$$
\begin{aligned}
& U:[0,1] \times X_{k_{0}}^{\infty} \rightarrow \Sigma^{+}, \\
& \left(t, \frac{1}{\widetilde{S}_{2}} \tilde{\delta}_{(x, \lambda)}\right) \rightarrow \frac{\frac{t}{H\left(y_{0}\right)} \tilde{\delta}_{\left(y_{0}, \lambda\right)}+\frac{(1-t)}{K(x)} \tilde{\delta}_{(x, \lambda)}}{\left\|\frac{t}{K\left(y_{0}\right)} \tilde{\delta}_{\left(y_{0}, \lambda\right)}+\frac{(1-t)}{K(x)} \tilde{\delta}_{(x, \lambda)}\right\|},
\end{aligned}
$$

$U$ is continuous and satisfies $U\left(0, \frac{1}{\widetilde{S}_{2}} \tilde{\delta}_{(x, \lambda)}\right)=\frac{1}{\tilde{S}_{2}} \tilde{\delta}_{(x, \lambda)} \in X_{k_{0}}^{\infty}$, and $U\left(1, \tilde{\delta}_{(x, \lambda)}\right)=\frac{1}{\tilde{S}_{2}} \tilde{\delta}_{\left(y_{0}, \lambda\right)}$, a fixed point of $X_{k_{0}}^{\infty}$. Furthermore, arguing as in [3], we derive that

$$
J\left(U\left(t, \frac{1}{\widetilde{S}_{2}} \tilde{\delta}_{(x, \lambda)}\right)\right) \leq \widetilde{S}_{2}\left(\frac{1}{K\left(y_{0}\right)}+\frac{1}{K(x)}\right)^{\frac{1}{2}}(1+o(x, \lambda))
$$


for each $\left(t, \frac{1}{S_{2}} \tilde{\delta}_{(x, \lambda)}\right) \in[0,1] \times X_{k_{0}}^{\infty}$. Here $o(x, \lambda)$ tends to zero when $\lambda$ tends to $+\infty$. Taking $\lambda$ large enough, since $\forall x \in X_{k_{0}}$ we have $K(x) \geq K\left(y_{k_{0}}\right)$, we get

$$
J\left(U\left(t, \frac{1}{\widetilde{S}_{2}} \tilde{\delta}_{(x, \lambda)}\right)\right) \leq c_{1}+\varepsilon, \quad \forall\left(t, \frac{1}{\widetilde{S}_{2}} \tilde{\delta}_{(x, \lambda)}\right) \in[0,1] \times X_{k_{0}}^{\infty}
$$

Therefore the contraction $U$ is performed under the level $c_{1}+\varepsilon$ and $X_{k_{0}}^{\infty}$ is then contractible in $J^{c_{1}+\varepsilon}$. Hence claim (17) follows. Let

$$
\theta\left(X_{k_{0}}^{\infty}\right):=U\left([0,1] \times X_{k_{0}}^{\infty}\right)
$$

Now, we use the gradient flow of $(-\partial J)$ to deform $\theta\left(X_{k_{0}}^{\infty}\right)$. Since $\theta\left(X_{k_{0}}^{\infty}\right) \subset J^{c_{1}+\varepsilon}$ and the only critical points at infinity of $J$ in $J^{c_{1}+\varepsilon}$ are $\left(y_{j}\right)_{\infty}, 0 \leq j \leq k_{0}$, by using a deformation lemma, see Proposition 7.24 and Theorem 8.2 of [39], we get

$$
\theta\left(X_{k_{0}}^{\infty}\right) \simeq X_{k_{0}}^{\infty} \cup \bigcup_{w<\theta\left(X_{k_{0}}^{\infty}\right)} W_{u}(w)
$$

where $w$ is a solution of (3) dominated by $\theta\left(X_{k_{0}}^{\infty}\right)$. Here $\simeq$ denotes retracts by deformation.

Now observe that it follows from the above deformation retract that problem (3) has necessarily a solution. Otherwise it follows from (18) that

$$
\theta\left(X_{k_{0}}^{\infty}\right) \simeq X_{k_{0}}^{\infty}
$$

Let $M$ be a $c w$ complex in dimension $k$, it is well known (see [40]) that the Euler-Poincaré characteristic of $M, \chi(M)$ is given by the following:

$$
\chi(M)=\sum_{r=0}^{k}(-1)^{r} n(r)
$$

where $n(r)$ is the number of cells of dimension $r$ in $M$. We apply this to our situation. By construction $X_{k_{0}}^{\infty}$ is a finite $c w$ complex, where the cells of dimension an integer $r$ in $X_{k_{0}}^{\infty}$ are given by the unstable manifolds of the critical points at infinity $(y)_{\infty}$ such that $i(y)_{\infty}=r$. According to (20), we derive that

$$
\chi\left(X_{k_{0}}^{\infty}\right)=\sum_{y_{j} \in \mathcal{K}^{+}, 0 \leq j \leq k_{0}}(-1)^{i\left(y_{j}\right)_{\infty}}
$$

From another part, it is easy to see that $\chi\left(\theta\left(X_{k_{0}}^{\infty}\right)\right)=1$, since $\theta\left(X_{k_{0}}^{\infty}\right)$ is a contractible set. Thus, we derive from (19), taking the Euler characteristic of both sides that

$$
1=\sum_{y_{j} \in \mathcal{K}^{+}, 0 \leq j \leq k_{0}}(-1)^{i\left(y_{j}\right)_{\infty}} .
$$

Such an equality contradicts the assumption of Theorem 1.2.

Now, for generic $K$, it follows from the Sard-Smale theorem that all solutions of (3) are non-degenerate solutions; see [10]. We apply the Euler-Poincaré characteristic argument, 
we derive from (18) that

$$
1=\sum_{y_{j} \in \mathcal{K}^{+}, 0 \leq j \leq k_{0}}(-1)^{i\left(y_{j}\right)_{\infty}}+\sum_{w<\theta\left(X_{k_{0}}^{\infty}\right)}(-1)^{i(w)},
$$

where $i(w)$ denotes the Morse index of the solution $w$. It follows then that

$$
\left|1-\sum_{y_{j} \in \mathcal{K}^{+}, 0 \leq j \leq k_{0}}(-1)^{i\left(y_{j}\right)_{\infty}}\right| \leq \sharp S,
$$

where $S$ denotes the set of solutions of (3).

Remark 3.1 Here, we want to consider some situation where the result of [28] does not give solution to problem (3), but using Theorem 1.2 we derive that problem (3) admits a solution. For this, let $K: \mathbf{S}^{2} \rightarrow \mathbf{R}$ be a $C^{2}$ positive Morse function satisfying the (nd) condition such that $\mathcal{K}^{+}$is reduced to 3 points $y_{0}, y_{1}$ and $y_{2}$ with $K\left(y_{0}\right) \geq K\left(y_{1}\right) \geq K\left(y_{2}\right) \cdot y_{0}$ is the absolute maximum of $K$, so $\operatorname{ind}\left(K, y_{0}\right)=2$. Assume also that $\operatorname{ind}\left(K, y_{1}\right) \neq \operatorname{ind}\left(K, y_{2}\right) \in$ $\{1,2\}$ and

$$
\frac{1}{K\left(y_{2}\right)}>\frac{1}{K\left(y_{0}\right)}+\frac{1}{K\left(y_{1}\right)} .
$$

It is easy to see that

$$
\sum_{y \in \mathcal{K}^{+}}(-1)^{\operatorname{ind}(K, y)}=1
$$

However, we have

$$
\begin{gathered}
\operatorname{Max}_{k \in\left(\mathbf{A}_{1}\right)}\left|1-\sum_{\substack{y_{j} \in \mathcal{K}^{+} \\
0 \leq j \leq k}}(-1)^{\operatorname{ind}\left(K, y_{j}\right)}\right| \\
=\left|1-\sum_{\substack{0 \leq \mathcal{K}^{+} \\
0 \leq j \leq 1}}(-1)^{\operatorname{ind}\left(K, y_{j}\right)}\right| \neq 0,
\end{gathered}
$$

so by Theorem 1.2 we derive the existence of a solution of problem (3).

\subsection{Proof of Theorem 1.3}

Let $\varepsilon \in \mathbf{R}, K_{\varepsilon}=1+\varepsilon K_{0}$ and $J_{\varepsilon}$ be the associated variational problem. So, for $u \in \Sigma^{+}$, we have

$$
J_{\varepsilon}(u)=\frac{\|u\|^{2}}{\left(\int_{\mathbf{S}^{n}} K_{\varepsilon}|u|^{\frac{2 n}{n-1}} d \sigma_{g_{0}}\right)^{\frac{n-1}{n}}} .
$$

Let us observe that in the case of the unit ball, in view of uniqueness result of $\mathrm{Li}$ and Zhu [36], a solution of (3) cannot be achieved as a minimum of $J_{\varepsilon}$, unless for $\varepsilon=0$. In this case, the functional $J_{0}$ possesses a $n$-dimensional manifold $Z$ of critical points, given by

$$
Z=\left\{\tilde{\delta}_{(a, \lambda)}, a \in \mathbf{S}^{n}, \lambda>0\right\} .
$$


Let $\widetilde{S}_{n}$ be the best constant of Sobolev associated to the Sobolev trace embedding $H^{1}\left(S^{n}\right) \hookrightarrow L^{\frac{2 n}{n-1}}\left(\mathbf{S}^{n}\right)$, it is easy to see that for every $\tilde{\delta}_{(a, \lambda)} \in Z$, we have

$$
J_{0}\left(\tilde{\delta}_{(a, \lambda)}\right)=\widetilde{S}_{n}=\inf _{u \in \Sigma^{+}} J_{0}(u)
$$

Given $a, b \in \mathbf{R}$, we set

$$
J_{\varepsilon}^{a}=\left\{u \in \Sigma^{+}, J_{\varepsilon}(u) \leq a\right\}
$$

and

$$
J_{\varepsilon b}^{a}=\left\{u \in \Sigma^{+}, b \leq J_{\varepsilon}(u) \leq a\right\} .
$$

We have the following lemma.

Lemma 3.2 Let $\eta>0$, for $|\varepsilon|$ sufficiently small, we have

$$
J_{\varepsilon}^{\widetilde{S}_{n}+\eta} \subset J_{0}^{\widetilde{S}_{n}+2 \eta} \subset J_{\varepsilon}^{\widetilde{S}_{n}+3 \eta}
$$

Proof Let $u \in \Sigma^{+}$, we have

$$
\begin{aligned}
J_{\varepsilon}(u) & =\frac{1}{\left(\int_{\mathbf{S}^{n}}|u|^{\frac{2 n}{n-1}} d x+\varepsilon \int_{\mathbf{S}^{n}} K_{0}|u|^{\frac{2 n}{n-1}} d x\right)^{\frac{n-1}{n}}} \\
& =J_{0}(u) \frac{1}{\left(1+\varepsilon\left(\int_{\mathbf{S}^{n}}|u|^{\frac{2 n}{n-1}} d x\right)^{-1} \int_{\mathbf{S}^{n}} K_{0}|u|^{\frac{2 n}{n-1}} d x\right)^{\frac{n-1}{n}}} .
\end{aligned}
$$

Using the fact that $K_{0}$ is bounded on $\mathbf{S}^{n}$, we derive that

$$
J_{\varepsilon}(u)=J_{0}(u)(1+O(\varepsilon))
$$

where $O(\varepsilon)$ is independent of $u$, tends to zero when $\varepsilon$ tends to zero. Hence the lemma follows.

Now let $\left(y_{1}, \ldots, y_{q}\right)_{\infty}$ a critical point at infinity of $q$-masses. It is easy to see that the level of $J_{\varepsilon}$ at $\left(y_{1}, \ldots, y_{q}\right)_{\infty}$ goes to $q \widetilde{S}_{n}$, when $\varepsilon$ tends to zero. Since

$$
c_{\infty}^{\varepsilon}\left(y_{1}, \ldots, y_{q}\right)_{\infty}=\widetilde{S}_{n}\left(\sum_{i=1}^{q} \frac{1}{K_{\varepsilon}\left(y_{i}\right)^{\frac{n-1}{2}}}\right)^{\frac{2}{n}} .
$$

Now, let $\eta=\frac{\widetilde{S}_{n}}{4}$, we can therefore assume that $|\varepsilon|$ is so small that all the critical points at infinity of $J_{\varepsilon}$ of two masses or more are above the level $\widetilde{S}_{n}+3 \eta$, and the critical points at infinity of one mass are below $\widetilde{S}_{n}+\eta$. Therefore,

$$
\text { the functional } J_{\varepsilon} \text { has no critical points at infinity in } \int_{\varepsilon\left(\widetilde{S}_{n}+\eta\right)}^{\widetilde{S}_{n}+3 \eta} \text {. }
$$


Arguing by contradiction and assume that (3) has no solution. It follows from (21) that

$$
J_{\varepsilon}^{\widetilde{S}_{n}+3 \eta} \simeq J_{\varepsilon}^{\widetilde{S}_{n}+\eta}
$$

where $\simeq$ denotes retracts by deformation. Using Lemma 3.2, we derive that

$$
J_{0}^{\widetilde{S}_{n}+2 \eta} \simeq J_{\varepsilon}^{\widetilde{S}_{n}+\eta}
$$

Now we claim that

$$
J_{\varepsilon}^{\widetilde{S}_{n}+\eta} \text { is a contractible set. }
$$

Indeed, from (23), it is sufficient to prove that $J_{0}^{\widetilde{S}_{n}+2 \eta}$ contractible set.

Let $u_{o} \in J_{0}^{\widetilde{S}_{n}+2 \eta}$, we solve

$$
\left\{\begin{array}{l}
\frac{\partial u}{\partial s}=-\partial J_{0}(u) \\
u(0)=u_{o}
\end{array}\right.
$$

Let $u\left(s, u_{0}\right)$ be the solution for $s>0$. Using the results of [38], concerning the Yamabe functional on $\mathbf{S}^{n}$, which is similar to our function $J_{0}$, we know that the Palais-Smale condition is satisfied for the above differential equation, up to $s=+\infty$. When $s$ tends to $+\infty, u\left(s, u_{0}\right)$ converges to a single mass in $Z$, thus

$$
J_{0}^{\widetilde{S}_{n}+2 \eta} \simeq Z
$$

We drive then that $J_{0}^{\widetilde{S}_{n}+2 \eta}$ is contractible since $Z$ is a contractible set. Hence our claim follows.

Now let $k_{0}$ be the integer where the

$$
\max _{k \in\left(\mathbf{A}_{2}\right)}\left|1-\sum_{y \in \mathcal{K}^{+}, i(y)_{\infty} \leq k}(-1)^{i(y)_{\infty}}\right|
$$

is achieved. Here $i(y)_{\infty}=n-1-\operatorname{ind}(K, y)$ is the Morse index of $(y)_{\infty}$. Let

$$
X_{k_{0}}^{\infty}=\bigcup_{y \in \mathcal{K}^{+}, i(y)_{\infty} \leq k_{0}} W_{u}^{\infty}(y)_{\infty} .
$$

$X_{k_{0}}^{\infty}$ is a stratified set of dimension at most $k_{0}$ in $J_{\varepsilon} \widetilde{S}_{n}+\eta$, without loss of generality we can assume that $X_{k_{0}}^{\infty}$ is of dimension $k_{0}$. Observe that $X_{k_{0}}^{\infty}$ is contractible in $J_{\varepsilon} \widetilde{S}_{n+\eta}$, since $\widetilde{S}_{\varepsilon}+\eta$ is a contractible set. More precisely there exists

$$
\begin{aligned}
& h:[0,1] \times X_{k_{0}}^{\infty} \rightarrow J_{\varepsilon}^{\widetilde{S}_{n+\eta}}, \\
& (t, u) \rightarrow h(t, u)
\end{aligned}
$$

where $h(0, u)=u, h$ is continuous and $h(1, u)=\tilde{u}$ a fixed point in $J_{\varepsilon} \widetilde{S}_{n}+\eta$.

Let $\theta\left(X_{k_{0}}^{\infty}\right)=h\left([0,1] \times X_{k_{0}}^{\infty}\right) . \theta\left(X_{k_{0}}^{\infty}\right)$ is a contractible stratified set of dimension $k_{0}+1$. Now, we use the gradient flow of $(-\partial J)$ to deform $\theta\left(X_{k_{0}}^{\infty}\right)$. By transversally arguments we 
can assume that the deformation avoids all critical points at infinity having their Morse indices greater or equal to $k_{0}+2$. Using the fact that $\theta\left(X_{k_{0}}^{\infty}\right) \subset J_{\varepsilon}^{\widetilde{S}_{n}+\eta}$, we get

$$
\theta\left(X_{k_{0}}^{\infty}\right) \simeq \bigcup_{y \in \mathcal{K}^{+}, i(y)_{\infty} \leq k_{0}+1} W_{u}^{\infty}(y)_{\infty}
$$

Since $k_{0}$ satisfying $\left(\mathbf{A}_{2}\right)$, there are no critical point at infinity $(y)_{\infty}, y \in \mathcal{K}^{+}$of index $k_{0}+1$, we therefore have

$$
\theta\left(X_{k_{0}}^{\infty}\right) \simeq X_{k_{0}}^{\infty}
$$

Thus, we derive from (27), taking the Euler characteristic of both sides that

$$
1=\sum_{y \in \mathcal{K}^{+}, i(y)_{\infty} \leq k_{0}}(-1)^{i(y)_{\infty}} .
$$

Such equality contradicts the assumption of Theorem 1.3. This completes the proof of Theorem 1.3.

\section{A general existence result}

In the last part of this paper, we give a generalization of Theorem 1.2. Namely instead of assuming that $\sigma=\frac{1}{2}$ and $n=2$, we assume that $\sigma \in(0,1)$ and $2 \leq n<2+2 \sigma$. Following the same scheme as Theorem 1.2, we state here an existence result to the following problem:

$$
P_{\sigma} u=c_{n, \sigma} K u^{\frac{n+2 \sigma}{n-2 \sigma}}, \quad u>0, \text { on } \mathbf{S}^{n},
$$

where

$$
P_{\sigma}=\frac{\Gamma\left(B+\frac{1}{2}+\sigma\right)}{\Gamma\left(B+\frac{1}{2}-\sigma\right)}, \quad B=\sqrt{-\Delta_{g}+\left(\frac{n-1}{2}\right)^{2}},
$$

$c_{n, \sigma}=P_{\sigma}(1), \Gamma$ is the gamma function and $\Delta_{g}$ is the Laplace-Beltrami operator on $\left(\mathbf{S}^{n}, g\right)$. We are now ready to state the following existence result.

Theorem 4.1 Let $K \in C^{1}\left(\mathbf{S}^{n}\right)$, be a positive function satisfying (nd) condition with $2 \leq n<$ $2+2 \sigma$ and $\sigma \in(0,1)$. If

$$
\operatorname{Max}_{k \in\left(\mathbf{A}_{1}\right)}\left|1-\sum_{\substack{y_{j} \in \mathcal{K}^{+} \\ 0 \leq j \leq k}}(-1)^{\operatorname{ind}\left(K, y_{j}\right)}\right| \neq 0,
$$

then (28) has at least one solution. Here, $\mathcal{K}^{+}=\left\{y \in \mathbf{S}^{n}, \nabla K(y)=0,-\Delta K(y)>0\right\}$. Moreover, for generic $K$ we have

$$
\sharp S \geq \operatorname{Max}_{k \in\left(\mathbf{A}_{1}\right)}\left|1-\sum_{\substack{y_{j} \in \mathcal{K}^{+} \\ 0 \leq j \leq k}}(-1)^{\operatorname{ind}\left(K, y_{j}\right)}\right|,
$$

where $S$ denotes the set of solutions of $(28)$. 
Proof The proof goes along with the proof of Theorem 1.2, therefore we will only sketch the differences. Keeping the notation of the proof of Theorem 1.2 and regarding the expansion of the functional $J$, we observe that the strong interaction of the bubbles in dimensions $n<2+2 \sigma$ forces all blow up points to be single. Thus, the only critical points at infinity associated to problem (28) correspond to

$$
(y)_{\infty}:=\frac{1}{K(y)^{\frac{n-2 \sigma}{n}}} \delta_{(y, \infty)}, \quad y \in \mathcal{K}^{+} .
$$

The Morse index of such critical point at infinity $(y)_{\infty}$ is: $i(y)_{\infty}=n-\operatorname{ind}(K, y)$. Now the remainder of the proof is identical to the proof of Theorem 1.2.

\section{Competing interests}

The authors declare that they have no competing interests.

\section{Authors' contributions}

All authors contributed equally and significantly in writing this paper. All authors read and approved the final manuscript.

\section{Author details}

${ }^{1}$ Department of Mathematics, King Abdulaziz University, Jeddah, Saudi Arabia. ${ }^{2}$ Department of Mathematics, Faculty of Sciences of Sfax, Sfax University, Soukra Road, Sfax, 3018, Tunisia.

\section{Acknowledgements}

We express our gratitude to the referees for their valuable criticisms of the manuscript and for helpful suggestions.

Received: 21 November 2013 Accepted: 21 July 2014 Published online: 26 September 2014

\section{References}

1. Graham, C, Jenne, R, Mason, LJ, Sparling, GAJ: Conformally invariant powers of the Laplacian. I. Existence. J. Lond. Math. Soc. 46, 557-565 (1992)

2. Bahri, A, Coron, JM: The scalar curvature problem on the standard three dimensional spheres. J. Funct. Anal. 95 106-172 (1991)

3. Ben Mahmoud, R, Chtioui, H: Existence results for the prescribed scalar curvature on $S^{3}$. Ann. Inst. Fourier 61, 971-986 (2011)

4. Ben Mahmoud, R, Chtioui, H: Prescribing the scalar curvature problem on higher-dimensional manifolds. Discrete Contin. Dyn. Syst. 32, 1857-1879 (2012)

5. Chang, S-YA, Gursky, MJ, Yang, PC: The scalar curvature equation on 2 and 3 spheres. Calc. Var. 1, $205-229$ (1993)

6. Chen, CC, Lin, CS: Prescribing the scalar curvature on $S^{n}$. I. A priori estimates. J. Differ. Geom. 57, 67-171 (2001)

7. Chtioui, H: Prescribing the scalar curvature problem on three and four manifolds. Adv. Nonlinear Stud. 3, 457-470 (2003)

8. Chtioui, H, Ben Mahmoud, R, Abuzaid, DA: Conformal transformation of metrics on the $n$-sphere. Nonlinear Anal. TMA 82, 66-81 (2013)

9. Li, Y: Prescribing scalar curvature on $S^{n}$ and related topics. Part I. J. Differ. Equ. 120, 319-410 (1995)

10. Schoen, R, Zhang, D: Prescribed scalar curvature on the $n$-sphere. Calc. Var. Partial Differ. Equ. 4, 1-25 (1996)

11. Abdelhedi, W, Chtioui, $\mathrm{H}$ : On the prescribed Paneitz curvature problem on the standard spheres. Adv. Nonlinear Stud. 6, 511-528 (2006)

12. Abdelhedi, W: On a fourth-order elliptic equation involving the critical Sobolev exponent: the effect of the graph topology. Nonlinear Anal. TMA 82, 82-99 (2013)

13. Al-Ghamdi, MA, Chtioui, H, Rigane, A: Existence of conformal metrics with prescribed Q-curvature. Abstr. Appl. Anal. 2013, Article ID 568245 (2013)

14. Bensouf, A, Chtioui, H: Conformal metrics with prescribed Q-curvature on $S^{n}$. Calc. Var. Partial Differ. Equ. 41, 455-481 (2011)

15. Chtioui, H, Rigan, A: On the prescribed Q-curvature problem on $S^{n}$. J. Funct. Anal. 261, 2999-3043 (2011)

16. Djadli, Z, Hebey, E, Ledoux, M: Paneitz-type operators and applications. Duke Math. J. 104, 129-169 (2000)

17. Djadli, Z, Malchiodi, A, Ahmedou, MO: Prescribing a fourth order conformal invariant on the standard sphere. Part I: a perturbation result. Commun. Contemp. Math. 4, 375-408 (2002)

18. Djadli, Z, Malchiodi, A, Ahmedou, MO: Prescribing a fourth order conformal invariant on the standard sphere. Part II: blow up analysis and applications. Ann. Sc. Norm. Super. Pisa 5, 387-434 (2002)

19. Wei, J, Xu, X: Prescribing Q-curvature problem on $S^{n}$. J. Funct. Anal. 257, 1995-2023 (2009)

20. Chang, S-YA, Gonzalez, M: Fractional Laplacian in conformal geometry. Adv. Math. 226, 1410-1432 (2011)

21. Graham, CR, Zworski, M: Scattering matrix in conformal geometry. Invent. Math. 152, 89-118 (2003)

22. Caffarelli, L, Silvestre, L: An extension problem related to the fractional Laplacian. Commun. Partial Differ. Equ. 32, 1245-1260 (2007)

23. Gonzalez, M, Qing, J: Fractional conformal Laplacians and fractional Yamabe problems. Anal. PDE 6, 1535-1576 (2013) 
24. Gonzalez, M, Mazzeo, R, Sire, Y: Singular solutions of fractional order conformal Laplacians. J. Geom. Anal. 22, 845-863 (2012)

25. Qing, J, Raske, D: On positive solutions to semilinear conformally invariant equations on locally conformally flat manifolds. Int. Math. Res. Not. 2006, Article ID 94172 (2006)

26. Xiong, TJJ: A fractional Yamabe flow and some applications (in press). arXiv:1110.5664V1

27. Stein, EM: Singular Integrals and Differentiability Properties of Functions. Princeton Mathematical Series, vol. 30. Princeton University Press, Princeton (1970)

28. Abdelhedi, W, Chtioui, H: On a Nirenberg type problem involving the square root of the Laplacian. J. Funct. Anal. 265 2937-2955 (2013)

29. Brandle, C, Colorado, E, de Pablo, A, Sanchez, U: A concave-convex elliptic problem involving the fractional Laplacian. Proc. R. Soc. Edinb., Sect. A 143, 39-71 (2013)

30. Cabré, $X$, Sire, Y: Nonlinear equations for fractional Laplacians, I: regularity, maximum principles, and Hamiltonian estimates. Ann. Inst. Henri Poincaré, Anal. Non Linéaire 31, 23-53 (2011)

31. Cabré, $X$, Tan, J: Positive solutions of nonlinear problems involving the square root of the Laplacian. Adv. Math. 224 2052-2093 (2010)

32. Capella, A, Davila, J, Dupaigne, L, Sire, Y: Regularity of radial extremal solutions for some non-local semilinear equations. Commun. Partial Differ. Equ. 8, 1353-1384 (2011)

33. Stinga, PR, Torrea, JL: Extension problem and Harnack's inequality for some fractional operators. Commun. Partial Differ. Equ. 35, 2092-2122 (2010)

34. Stuwe, M: A global compactness result for elliptic boundary value problem involving limiting nonlinearities. Math. Z. 187, 511-517 (1984)

35. Bahri, A: An invariant for Yamabe-type flows with applications to scalar curvature problems in high dimensions. A celebration of J.F. Nash Jr. Duke Math. J. 81, 323-466 (1996)

36. Li, Y, Zhu, M: Uniqueness theorems through the method of moving spheres. Duke Math. J. 80, $383-417$ (1995)

37. Brezis, H, Coron, JM: Convergence of solutions of $\mathrm{H}$-systems or how to blow bubbles. Arch. Ration. Mech. Anal. 89 21-56 (1985)

38. Bahri, A: Critical Point at Infinity in Some Variational Problems. Pitman Research Notes in Mathematics Series, vol. 182 (1989)

39. Bahri, A, Rabinowitz, P: Periodic orbits of Hamiltonian systems of three body type. Ann. Inst. Henri Poincaré, Anal. Non Linéaire 8, 561-649 (1991)

40. Hatcher, A: Algebraic Topology. Cambridge University Press, Cambridge (2002)

doi:10.1186/s13661-014-0187-2

Cite this article as: Al-Ghamdi et al.: Topological arguments for an elliptic equation involving the fractional

Laplacian. Boundary Value Problems 2014 2014:187.

\section{Submit your manuscript to a SpringerOpen ${ }^{\odot}$ journal and benefit from:}

- Convenient online submission

Rigorous peer review

- Immediate publication on acceptance

- Open access: articles freely available online

- High visibility within the field

- Retaining the copyright to your article 